\title{
Anatomical Courses of Lateral Antebrachial and Medial Antebrachial Cutaneous Nerves: A Cadaveric Study
}

\author{
Ye-Ji Kwon ${ }^{a}$ \\ Jae-Gyum Kim ${ }^{\mathrm{a}}$ \\ Dasom Kim ${ }^{\mathrm{b}}$ \\ Im Joo Rhyu ${ }^{\mathrm{b}}$ \\ Byung-Jo Kim ${ }^{\mathrm{a}}$ \\ aDepartment of Neurology, \\ Korea University Medical Center \\ Seoul, Korea \\ bDepartment of Anatomy, \\ Korea University College of Medicine, \\ Seoul, Korea
}

Received March 11, 2019

Revised March 26, 2019

Accepted March 28, 2019

\section{Correspondence}

Byung-Jo Kim, MD, PhD

Department of Neurology,

Korea University Medical Center,

73 Goryeodae-ro, Seongbuk-gu,

Seoul 02841, Korea

Tel +82-2-920-5510

Fax +82-2-925-2472

E-mail nukbj@korea.ac.kr
Dear Editor,

Correctly interpreting the findings of nerve conduction studies (NCS) in cases of focal lesions of the peripheral nerves requires a detailed understanding of the anatomy. In contrast to the well-documented motor nerve variations, little detailed information is available on the variations of sensory nerves, particularly those innervating the forearm. ${ }^{1}$ Sensory NCS of the lateral antebrachial cutaneous nerve $(\mathrm{LABCN})$ and the medial antebrachial cutaneous nerve $(\mathrm{MABCN})$ are commonly conducted to evaluate nerve injuries of the upper extremities. The technical errors that are common in the NCS of these nerves mean that more precise techniques need to be applied compared with other nerves. ${ }^{2}$ We aimed to address technical considerations while conducting NCS through fresh cadaveric dissections of these cutaneous nerves.

We examined 18 arms of 9 fresh cadavers (3 females and 6 males aged 60-98 years at death) in the anatomical position. None of the cadavers had any history of trauma or neuromuscular disease. The dissection started at the elbow to find the superficially located nerves, which were carefully traced to detect the target nerves. After exposing both nerves, we measured the distances between the nerves and common landmarks-the tips of the lateral epicondyle (LE) and medial epicondyle (ME) for the LABCN and MABCN, respectively-at the level of the interepicondylar line (IEL). If there was more than one branch, we calculated the mean distance for each branch.

LABCNs emerged from the lateral border of the biceps brachii tendon and had a relatively consistent appearance when they passed the IEL (Fig. 1A). Three arms had two LABCN branches, and the other arms had only one branch at the level of the IEL (Fig. 1C). The distance from the $\mathrm{LE}$ was $3.53 \pm 0.85 \mathrm{~cm}$ (mean $\pm \mathrm{SD})$, corresponding to $33.20 \pm 8.37 \%$ of the distance to the IEL (Fig. 1B).

The MABCNs were more difficult to identify, and were not found at all in three arms. These nerves were thinner and exhibited a greater variety of courses and more distal branches. There were intrasubject differences in the course and branches between the right and left sides in seven of the nine cadavers. Among the $15 \mathrm{arms}$ in which MABCNs were found, 7 had 1 branch, 4 had 2 branches, 3 had 3 branches, and 1 had 4 branches at the level of IEL (Fig. 1C). The distance from the ME was $2.87 \pm 1.14 \mathrm{~cm}$, corresponding to $26.3 \pm$ $9.36 \%$ of the distance to the IEL.

Our NCS of the MABCN and LABCN employed the conventional method. ${ }^{3}$ Anatomical variations in peripheral nerves or nerves with tortuous courses in deep regions may result in the findings of NCS being misinterpreted in clinical practice. ${ }^{4,5}$ In the present study the LABCN was found approximately lateral one-third of IEL. This position was relatively consistent in most cases, and hence it might be an appropriate reference point for stimulation

@ This is an Open Access article distributed under the terms of the Creative Commons Attribution Non-Commercial License (https://creativecommons.org/licenses/by-nc/4.0) which permits unrestricted non-commercial use, distribution, and reproduction in any medium, provided the original work is properly cited. 

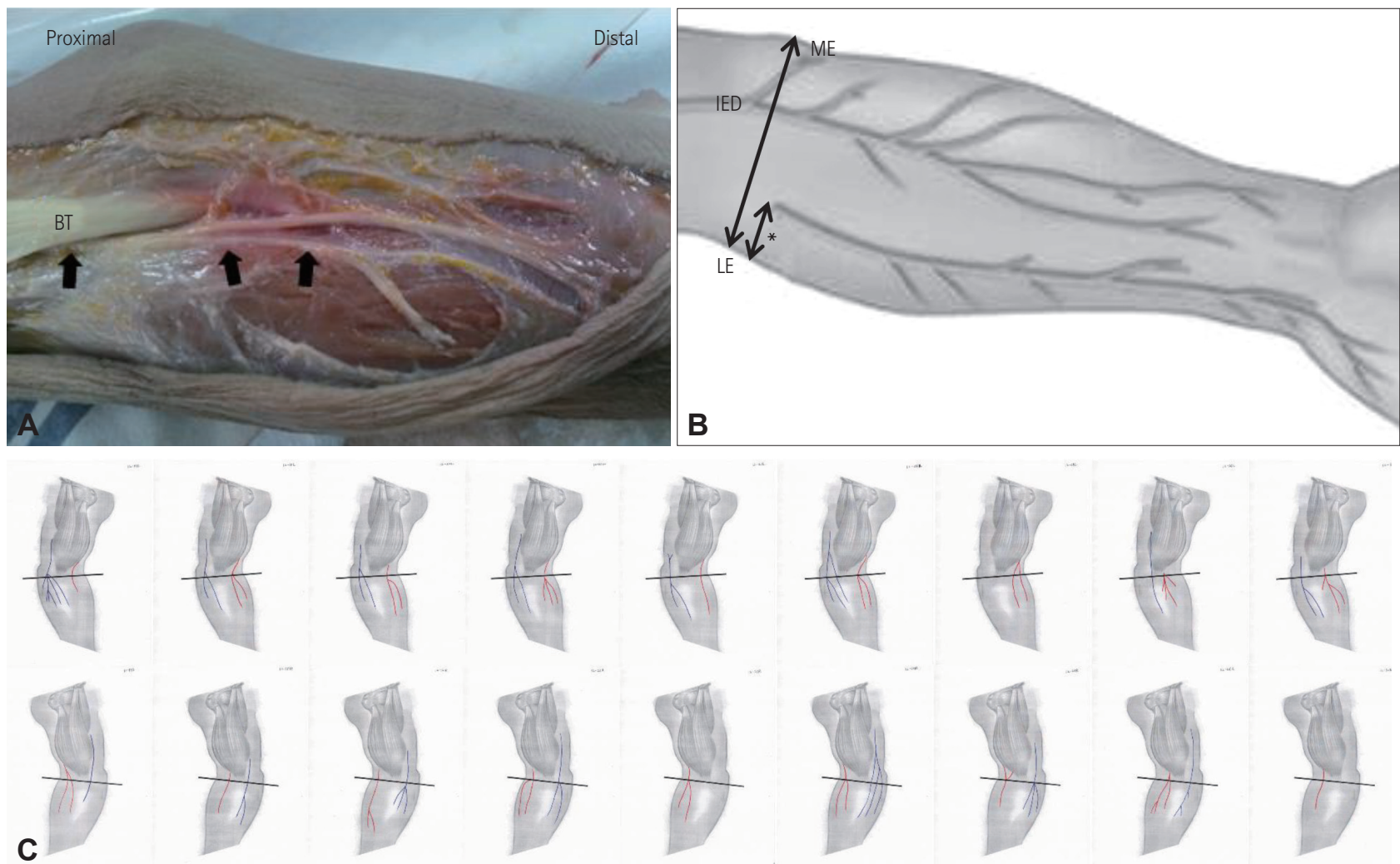

Fig. 1. A: Dissection of a right forearm in an anterior view showing the $L A B C N$ (arrows). B: The IED and distance from the $L A B C N$ to the $L E$ (asterisk). C: Schematic illustrations of medial antebrachial cutaneous nerves (blue lines) and LABCNs (red lines) at the elbow in the nine cadavers. BT: biceps brachii tendon, IED: interepicondylar distance, LABCN: lateral antebrachial cutaneous nerve, LE: lateral epicondyle, ME: medial epicondyle.

in NCS. We found most MABCNs more medial to the medial one third of IEL. The distal nerves were slender, became sparse, had more diverse courses, and their distal parts were divided into more than two branches. The findings of this study indicate that applying the conventional NCS method to the MABCN is challenging, and it is difficult to determine any nerve abnormality in only a single trial. It is therefore important to find the ideal stimulation and recording sites with side-to-side comparisons of amplitude and latency in several trials.

In conclusion, this study has revealed anatomical variations in the MABCN and LABCN that might result in erroneous NCS interpretations. If the conventional method provides uncertain results that are not consistent with the symptoms of the patient, adjusting the stimulation and recording sites should be considered. This approach might reduce technical errors and help to obtain more accurate results.

\section{Conflicts of Interest}

The authors have no potential conflicts of interest to disclose.

\section{REFERENCES}

1. Kimura J. Electrodiagnosis in diseases of nerve and muscle: principles and practice. 3rd ed. New York: Oxford University Press, 2001.

2. Izzo KL, Aravabhumi S, Jafri A, Sobel E, Demopoulos JT. Medial and lateral antebrachial cutaneous nerves: standardization of technique, reliability and age effect on healthy subjects. Arch Phys Med Rehabil 1985; 66:592-597.

3. Dumitru D, Amato AA, Zwarts MJ. Electrodiagnostic medicine. 2nd ed. Philadelphia: Hanley \& Belfus, 2002.

4. Koo YS, Cho CS, Kim BJ. Pitfalls in using electrophysiological studies to diagnose neuromuscular disorders. J Clin Neurol 2012;8:1-14.

5. Kim JG, Kim D, Seok HY, Kim Y, Yang KS, Rhyu IJ, et al. A method of radial nerve length measurement based on cadaveric investigation. Arch Phys Med Rehabil 2017;98:596-599. 